\title{
The $k$-step spatial sign covariance matrix
}

\author{
C. Croux - C. Dehon - A. Yadine
}

Received: 20 November 2009 / Revised: 13 April 2010 / Accepted: 24 April 2010 /

Published online: 11 June 2010

(C) The Author(s) 2010. This article is published with open access at Springerlink.com

\begin{abstract}
The Sign Covariance Matrix is an orthogonal equivariant estimator of multivariate scale. It is often used as an easy-to-compute and highly robust estimator. In this paper we propose a $k$-step version of the Sign Covariance Matrix, which improves its efficiency while keeping the maximal breakdown point. If $k$ tends to infinity, Tyler's M-estimator is obtained. It turns out that even for very low values of $k$, one gets almost the same efficiency as Tyler's M-estimator.
\end{abstract}

Keywords Breakdown point - Multivariate analysis · Principal components · Robust estimation · Spatial signs

Mathematics Subject Classification (2000) 62

C. Croux

Faculty of Business and Economics, K.U. Leuven, Louvain, Belgium

C. Croux ( $\otimes)$

Faculty of Economics and Business Administration, Tilburg University, Tilburg, The Netherlands e-mail: Christophe.Croux@econ.kuleuven.be

C. Dehon

Institut de Recherche en Statistique, ECARES, Université libre de Bruxelles, CP-114, Av. F.D. Roosevelt 50, 1050 Brussels, Belgium e-mail: cdehon@ulb.ac.be

A. Yadine

Institut de Recherche en Statistique, Université libre de Bruxelles,

Av. F.D. Roosevelt 50, 1050 Brussels, Belgium

e-mail: ayadine@hotmail.com 


\section{Introduction}

Let $X=\left\{X_{1}, X_{2}, \ldots, X_{n}\right\}$ be a set of $n$ multivariate observations, with each $X_{i}$ a vector of dimension $p$. These observations are a random sample from a distribution $F$. We assume that the distribution $F$ is elliptically symmetric with center $\mu$ and scatter matrix $\Sigma$. The density of $F$ can then be written as

$$
f_{\mu, \Sigma}(x)=\frac{1}{\operatorname{det}(\Sigma)^{1 / 2}} g\left((x-\mu)^{t} \Sigma^{-1}(x-\mu)\right),
$$

with $\operatorname{det}(\Sigma)$ the determinant of $\Sigma$ and $g$ a function taking positive values and scaled such that the density $f$ integrates to one. If the second moment of $F$ exists, then $\Sigma$ is a multiple of the covariance matrix. In this paper we focus on $F=N(0, \Sigma)$, but the obtained results extend to any elliptically symmetric distribution.

It is well known that the sample covariance matrix, while being the most efficient at normal distributions, is very vulnerable in presence of outliers. A very simple robust estimator of $\Sigma$ is the Sign Covariance Matrix (SCM), defined as

$$
\hat{\Sigma}_{S}(X)=\frac{1}{n} \sum_{i=1}^{n} \frac{\left(X_{i}-\hat{\mu}_{n}\right)\left(X_{i}-\hat{\mu}_{n}\right)^{t}}{\left\|X_{i}-\hat{\mu}_{n}\right\|^{2}} .
$$

This estimator is nothing else but the usual covariance matrix computed from the spatial signs of the observations, defined as

$$
U\left(X_{i}\right)=\frac{\left(X_{i}-\hat{\mu}_{n}\right)}{\left\|X_{i}-\hat{\mu}_{n}\right\|} .
$$

Since the spatial signs are bounded vectors, with unit norm, the SCM always remains bounded, indicating its robustness. The SCM and its usefulness in different applications are discussed in Locantore et al. (1999); Visuri et al. (2003); Sirkia et al. (2009); Oja (2010), among others. For the location estimator $\hat{\mu}_{n}$ in (2) we take the $L_{1}$-median, also called the spatial median, defined as

$$
\hat{\mu}=\underset{\mu}{\operatorname{argmin}} \frac{1}{n} \sum_{i=1}^{n}\left\|X_{i}-\mu\right\| .
$$

The $L_{1}$-estimator is highly robust, and as solution of a convex optimization problem very fast to compute.

A first contribution of this paper is that we formally show that the breakdown point of the SCM is the highest possible, namely $50 \%$. The breakdown point of an estimator is a standard measure of robustness, and gives the highest fraction of outliers the estimator can withstand. A formal definition is given in Sect. 2. At first sight one may think that the breakdown point of the SCM should be $100 \%$, since its norm is always bounded by one. However, breakdown may also occur if the estimator implodes, meaning that the smallest eigenvector of the SCM tends to zero. Implosion breakdown is important, since one often inverts scatter matrices, and a full rank of the scatter matrix estimator is desirable. 
A major drawback of the SCM is that it is only orthogonally equivariant. This means that $\hat{\Sigma}_{S}(A X)=A \hat{\Sigma}_{S}(X) A^{t}$ for any orthogonal matrix $A$, but not for any non-singular matrix $A$, which would imply affine equivariance. The lack of affine equivariance also results in a severe loss of statistical power when the true distribution deviates strongly from sphericity, as was shown in Croux et al. (2002). To increase the efficiency of the $\mathrm{SCM}$, we propose to perform a one-step update of the SCM,

$$
\hat{\Sigma}_{1}(X)=\frac{1}{n} \sum_{i=1}^{n} \frac{\left(X_{i}-\hat{\mu}_{n}\right)\left(X_{i}-\hat{\mu}_{n}\right)^{t}}{\left(X_{i}-\hat{\mu}_{n}\right)^{t} \hat{\Sigma}_{S}^{-1}\left(X_{i}-\hat{\mu}_{n}\right)} .
$$

More generally, the $k$-step SCM estimator is defined by

$$
\hat{\Sigma}_{k}(X)=\frac{1}{n} \sum_{i=1}^{n} \frac{\left(X_{i}-\hat{\mu}_{n}\right)\left(X_{i}-\hat{\mu}_{n}\right)^{t}}{\left(X_{i}-\hat{\mu}_{n}\right)^{t} \hat{\Sigma}_{k-1}^{-1}\left(X_{i}-\hat{\mu}_{n}\right)},
$$

for $k>1$. We will show that the $k$-step SCM estimator keeps the breakdown point of the initial SCM, but achieves a higher efficiency at non-spherical distributions. We stress that the location estimator $\hat{\mu}_{n}$ is not updated in (4) and (5). Focus in this paper is on the estimation of the scatter matrix $\Sigma$.

If $k$ tends to infinity, then the $k$-step SCM estimator converges to Tyler's M, an affine equivariant estimator. In fact, Tyler (1987) proposed an iterative algorithm to compute his estimator and proved its convergence. One has that $\hat{\Sigma}_{k}$ corresponds to the $k$ th step approximation of Tyler's M estimator. In this paper, we consider $\hat{\Sigma}_{k}$ as an estimator in its own right, being orthogonal equivariant and having good robustness properties. While Tyler's M-estimator has a breakdown point decreasing with the dimension, this does not hold for $\hat{\Sigma}_{k}$. So keeping $k$ fixed results in a high breakdown point, a property one looses by running the iterative algorithm to infinity.

The paper is organized as follows. Section 2 shows that the SCM and its $k$-step version have the maximal breakdown point property. Section 3 contains results on statistical efficiency. We derive an analytical expression for the asymptotic efficiency in the bivariate Gaussian case. Simulation results are presented in Sects. 4, and 5 contains the conclusions and limitations of this paper.

\section{Breakdown point}

In this section we compute the breakdown point of the SCM and the $k$-step version. We prove that they attain the highest possible breakdown point of $50 \%$. The location estimator used in definitions (2) and (5) is the $L_{1}$-median. The breakdown point of a multivariate location estimator $\hat{\mu}_{n}$ at the sample $X$ is defined as

$$
\varepsilon^{*}\left(\hat{\mu}_{n}, X\right)=\min _{1 \leq m \leq n}\left\{\frac{m}{n}: \sup _{X^{\prime}}\left\|\hat{\mu}_{n}(X)-\hat{\mu}_{n}\left(X^{\prime}\right)\right\|=+\infty\right\} .
$$

The supremum is taken over all possible corrupted collections $X^{\prime}$ that can be obtained by replacing any $m$ points $X_{1}, \ldots, X_{m}$ of $X$ by arbitrary values $X_{1}^{\prime}, \ldots, X_{m}^{\prime}$. Lopuhäa and Rousseeuw (1991) showed that the $L_{1}$-median has the largest possible breakdown 
point of any translation equivariant estimator:

$$
\varepsilon^{*}\left(\hat{\mu}_{n}, X\right)=\frac{\left\lfloor\frac{n+1}{2}\right\rfloor}{n} .
$$

The breakdown point of a multivariate scale estimator $\hat{\Sigma}$ at a data set $X$ is defined as the smallest fraction of outliers that can either take the largest eigenvalue over all bounds, or take the smallest eigenvalue arbitrarily close to 0 . Denote the ordered eigenvalues of any matrix $\Sigma$ by $\lambda_{1}(\Sigma) \geq \cdots \geq \lambda_{p}(\Sigma)$. This formal definition of the breakdown point is then

$$
\varepsilon^{*}(\hat{\Sigma}, X)=\min _{1 \leq m \leq n}\left\{\frac{m}{n}: \sup _{X^{\prime}} \max \left\{\lambda_{1}\left(\hat{\Sigma}\left(X^{\prime}\right)\right), \quad \lambda_{p}\left(\hat{\Sigma}\left(X^{\prime}\right)\right)^{-1}\right\}=+\infty\right\},
$$

where the supremum is taken over the same collections $X^{\prime}$ as before. We first show that the SCM estimator has the same breakdown point as the $L_{1}$-median. A proof of this proposition is given in the Appendix.

Proposition 1 Let $X$ be a sample of size $n$ such that no $\lfloor n / 2\rfloor+1$ points are contained in the same hyperplane. Then

$$
\varepsilon^{*}\left(\Sigma_{S}, X\right)=\left[\frac{n+1}{2}\right] / n .
$$

The $k$-step estimator keeps the breakdown point of the initial SCM estimator. The proof relies on the following identity

$$
\hat{\Sigma}_{k}(X)=\hat{\Sigma}_{k-1}(X)^{1 / 2} \hat{\Sigma}_{S}\left(\hat{\Sigma}_{k-1}^{-1 / 2}(X) X\right) \hat{\Sigma}_{k-1}(X)^{1 / 2},
$$

for any $k \geq 1$, and with $\hat{\Sigma}_{0}:=\hat{\Sigma}_{S}$. It is not difficult to show, combining (7) and Proposition 1, that $\hat{\Sigma}_{k}$ will inherit the breakdown point of $\hat{\Sigma}_{k-1}$. By induction we get that

Proposition 2 Let $X$ be a sample of size $n$ such that no $\lfloor n / 2\rfloor+1$ points are contained in the same hyperplane. Then

$$
\varepsilon^{*}\left(\Sigma_{k}, X\right)=\left[\frac{n+1}{2}\right] / n
$$

for every $k \leq 1$.

The above result holds for every fixed value of $k$. When we let $k$ tend to infinity, by iterating formula (5) up to convergence, the breakdown point is not maximal anymore and depends on the dimension. Dümbgen and Tyler (2005) showed that an upper bound for Tyler's M-estimator is given by $1 / p$, with $p$ the dimension. This finding provides some theoretical support for the results of Hettmansperger and Randles (2002), who said that Tyler's M-estimator has a 'practical' breakdown point of $50 \%$. 


\section{Asymptotic efficiencies}

In this section we study some aspects of the limiting distribution of the $k$-step SCM estimators. In particular we compute asymptotic efficiencies at the bivariate normal distribution, but the obtained results can be easily extended to other elliptically symmetric distributions. Let us first define the population quantities that are estimated. For a given distribution $F$, let

$$
T(F)=\underset{\mu}{\operatorname{argmin}} E_{F}[\|X-\mu\|]
$$

be the population version of the spatial median. The population counterpart of the $\mathrm{SCM}$ is

$$
\Sigma_{S}(F):=\Sigma_{0}(F)=E_{F}\left[\frac{(X-T(F))(X-T(F))^{t}}{(X-T(F))^{t}(X-T(F))}\right]
$$

and the functional $k$-step SCM is defined recursively as

$$
\Sigma_{k}(F)=E_{F}\left[\frac{(X-T(F))(X-T(F))^{t}}{(X-T(F))^{t} \Sigma_{k-1}^{-1}(F)(X-T(F))}\right],
$$

for $k \geq 1$. It is worth mentioning that also the SCM verifies (9), with $\Sigma_{-1}(F)=I_{p}$, the identity matrix. In the limit, for $k \rightarrow \infty$, we get the population version of Tyler's estimator, as solution of the equation

$$
\Sigma_{\infty}(F)=E_{F}\left[\frac{(X-T(F))(X-T(F))^{t}}{(X-T(F))^{t} \Sigma_{\infty}^{-1}(F)(X-T(F))}\right],
$$

where the constraint $\operatorname{Trace}\left(\Sigma_{\infty}(F)\right)=p$ is imposed to ensure a unique solution. Alternatively, one could use the constraint $\operatorname{det}\left(\Sigma_{\infty}(F)\right)=1$, see Paindaveine (2008).

A scatter matrix $\Sigma$ can always be decomposed as

$$
\Sigma=\lambda U D U^{t},
$$

where $U$ contains the eigenvectors of $\Sigma, D$ is a diagonal matrix containing the scaled eigenvalues of $\Sigma$, and $\lambda=\operatorname{det}(\Sigma)$. The size of the scatter matrix is then determined by $\lambda$, the matrix $U D U^{t}$ is the shape matrix while $U$ determines the orientation of the scatter matrix (Bensmail and Celeux 1996). Since all estimators we consider are at least orthogonal equivariant, we may assume without loss of generality that the scatter matrix $\Sigma$ of the distribution $F$ is diagonal. Using symmetry arguments, it is then immediate to check that $\Sigma_{k}(F)$ is also diagonal, for every $k \geq 0$. We thus have that the SCM and its $k$-step versions have the same orientation as the scatter matrix $\Sigma$, but the shape and the size will be different.

To compare the precision of the different $k$-step estimators in a meaningful way, we compare the asymptotic variances of their eigenvectors, who are all estimating the 
same quantity at elliptical model distributions. We only present results for the bivariate case $(p=2)$, to facilitate the exposition, and because it allows for an explicit expression of the asymptotic variances. The model distribution is then denoted by $F_{\gamma}$, having scatter matrix

$$
\Sigma=\left(\begin{array}{ll}
1 & 0 \\
0 & \gamma
\end{array}\right), \quad 0<\gamma<1
$$

Without loss of generality, we assume that the center of the model distribution is zero, so $T\left(F_{\gamma}\right)=0$. The eigenvectors have norm one, and are orthogonal, so it suffices to study the distribution of the second component of the first eigenvector of $\hat{\Sigma}_{k}$ (for $p=2$ ). Since the location and the scatter matrix estimator are asymptotically independent at elliptical models, the asymptotic variances of the eigenvectors do not depend on $T$.

\subsection{Asymptotic variance}

For $p=2$, the asymptotic variance of the second component of the first eigenvector of $\hat{\Sigma}_{k}$ at $F_{\gamma}$ equals

$$
A S V_{k}\left(F_{\gamma}\right)=\frac{1}{\left(\lambda_{k, 2}-\lambda_{k, 1}\right)^{2}} E\left[I F\left(X, \Sigma_{k, 12} ; F_{\gamma}\right)^{2}\right],
$$

see Croux and Haesbroeck (2000), Lemma 3. The computation of the influence function of the off-diagonal element of $\hat{\Sigma}_{k}$ is not difficult. Using standard influence function techniques, we get that the influence function of an off-diagonal element of the $k$-step SCM estimator at $x=\left(x_{1}, x_{2}\right)^{t}$ equals

$$
I F\left(x, \Sigma_{k, 12} ; F_{\gamma}\right)=\frac{x_{1} x_{2}}{x^{t} \Sigma_{k-1}^{-1}\left(F_{\gamma}\right) x}+c_{k-1} I F\left(x, \Sigma_{k-1,12} ; F_{\gamma}\right) \text {. }
$$

We have that

$$
c_{s}=2 E_{F_{\gamma}}\left[\frac{X_{1}^{2} X_{2}^{2}}{\left(X^{t} \Sigma_{s}\left(F_{\gamma}\right)^{-1} X\right)^{2}}\right] \frac{1}{\lambda_{s, 1} \lambda_{s, 2}},
$$

and

$$
\Sigma_{s}\left(F_{\gamma}\right)=\left(\begin{array}{cc}
\lambda_{s, 1} & 0 \\
0 & \lambda_{s, 1}
\end{array}\right)
$$

for every $s \geq 0$. Hence the influence function can be computed recursively from (14). The $I F$ for $k=0$, corresponding to the SCM, is given by

$$
I F\left(x, \Sigma_{0,12} ; F_{\gamma}\right)=\frac{x_{1} x_{2}}{\|x\|^{2}},
$$

and still obeys (14) if we set $c_{-1}=0$, and $\lambda_{-1,1}=\lambda_{-1,2}=1$, such that $\Sigma_{-1}\left(F_{\gamma}\right)=I$. 
Working out the recursive relation (14), combined with (13), results in

$$
A S V_{k}\left(F_{\gamma}\right)=\frac{1}{\left(\lambda_{k, 2}-\lambda_{k, 1}\right)^{2}} a_{k}^{t} B_{k} a_{k}
$$

with $a_{k}=\left(1, c_{k-1}, c_{k-1} c_{k-2}, \ldots, c_{k-1} c_{k-2}, \ldots, c_{-1}\right)^{t}$ a vector of length $k+1$, and $B_{k}$ a square matrix of size $k+1$, with elements

$$
\left(B_{k}\right)_{j_{1}, j_{2}}=E_{F_{\gamma}}\left[\frac{X_{1}^{2} X_{2}^{2}}{\left(X^{t} \Sigma_{k-j_{1}}\left(F_{\gamma}\right)^{-1} X\right)\left(X^{t} \Sigma_{k-j_{2}}\left(F_{\gamma}\right)^{-1} X\right)}\right]
$$

for $1 \leq j_{1}, j_{2} \leq k+1$, and for every $k \geq 0$. The vector $a_{k}$, the matrix $B_{k}$ and the scalar $c_{k}$ all depend on $\gamma$.

For the SCM, so $k=0$, the asymptotic variance was computed by Croux et al. (2002). At the normal distribution they obtain

$$
A S V_{0}\left(F_{\gamma}\right)=\frac{\sqrt{\gamma}}{2(1-\sqrt{\gamma})^{2}}
$$

Below we show how analytic expressions for the asymptotic variance for $k \geq 1$ can be obtained.

\subsection{Calculus}

In this section we provide some lemmas allowing us to compute the $A S V_{k}$ in (16). For computing the constant vector $a_{k}$ and the matrix $B_{k}$ in (16), we need to evaluate quantities of the form

$$
\Psi(a, b)=E_{0}\left[\frac{X_{1}^{2} X_{2}^{2}}{\left(X_{1}^{2}+a X_{2}^{2}\right)\left(X_{1}^{2}+b X_{2}^{2}\right)}\right],
$$

with $a, b>0$. Furthermore, we also need the eigenvalues of $\Sigma_{k}\left(F_{\gamma}\right)$. The first lemma gives an expression for these eigenvalues. The second lemma gives two formulas allowing to compute analytically the asymptotic variances at the normal distribution. The results can be obtained by straightforward calculus, and details can be found in the Ph.D. manuscript of Yadine (2006).

Lemma 1 Let $\lambda_{k, 1}$ and $\lambda_{k, 2}$ be the eigenvalues of $\Sigma_{k}\left(F_{\gamma}\right)$, wiht $0<\gamma<1$. Then the following recursion relations hold:

$$
\lambda_{k, 1}=\lambda_{k-1,1}\left(1-\gamma \frac{\lambda_{k-1,1}}{\lambda_{k-1,2}} \varphi\left(\gamma \frac{\lambda_{k-1,1}}{\lambda_{k-1,2}}\right)\right)
$$


and

$$
\lambda_{k, 2}=\gamma \lambda_{k-1,1} \varphi\left(\gamma \frac{\lambda_{k-1,1}}{\lambda_{k-1,2}}\right),
$$

with

$$
\varphi(a)=E_{F_{1}}\left[\frac{X_{1}^{2}}{X_{1}^{2}+a X_{2}^{2}}\right],
$$

for any $a>0$, and $k \geq 0$. Recall that $\lambda_{-1,1}=\lambda_{-1,2}=1$.

Lemma 2 For $F_{\gamma}$ a normal distribution, we have

$$
\varphi(a)=\frac{1}{\sqrt{a}(1+\sqrt{a})}
$$

and

$$
\Psi(a, b)=\frac{1}{(\sqrt{a}+\sqrt{b})(1+\sqrt{a})(1+\sqrt{b})},
$$

for all $a, b>0$.

\subsection{Efficiency of $k$-step estimators for bivariate Gaussian distributions}

At the normal distribution, the most efficient estimator for $\Sigma$ is the sample covariance matrix, being the maximum likelihood estimator. It is not difficult to verify that

$$
A S V_{M L}\left(F_{\gamma}\right)=\frac{\gamma}{(1-\gamma)^{2}}
$$

We then define the efficiency of the $k$-step SCM as

$$
\operatorname{Eff}_{k}\left(F_{\gamma}\right)=\frac{A S V_{M L}\left(F_{\gamma}\right)}{A S V_{k}\left(F_{\gamma}\right)}
$$

for $k \geq 0$. Using the results of Sects. 3.1 and 3.2 the efficiency can be computed analytically at Gaussian distributions. For example, for the one-step SCM we have

$$
\operatorname{Eff}_{1}\left(F_{\gamma}\right)=\frac{2 \gamma^{1 / 4}\left(1-\sqrt{\gamma} \gamma^{1 / 4}\right)^{2}\left(1+\gamma^{1 / 4}\right)^{2}}{(1-\gamma)^{2}\left[\left(1+\gamma^{1 / 4}\right)^{2}+5 \gamma^{1 / 4}\right]}
$$

In Fig. 1 we plot the efficiencies of the $k$-step SCM estimator for different values of $k$, as a function of $\gamma$, where $\gamma$ is the ratio between the smallest and the largest eigenvalue of the model covariance matrix. We see that the efficiencies converge very 


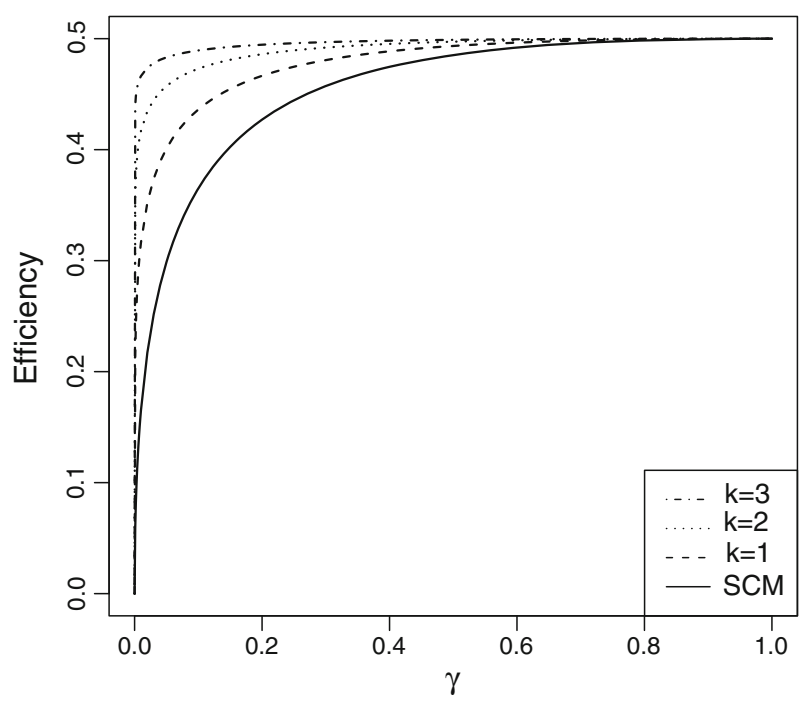

Fig. 1 Efficiencies of the SCM and its $k$-step version at the bivariate normal distribution, as a function of $\gamma$, the ratio between the smallest and the largest eigenvalue of the model covariance matrix. The efficiency of Tyler's M-estimator is constant and equal to 0.5 . We see that, for increasing $k$, there is fast convergence to this value

quickly to the value 0.5 , when $k$ tends to infinity. This limiting value corresponds to the efficiency of Tyler's M-estimator, being $k /(k+2)=0.5$ (Tyler 1987; Frahm 2009). This value does not depend on the value of $\gamma$ since Tyler's $M$ shape is an affine equivariant estimator (Ollila et al. 2002). For the $k$-step SCM we observe a loss of efficiency if we deviate strongly from spherical distribution, i.e. when $\gamma$ is close to zero and where $\Sigma_{\gamma}$ is close to singular The surprising finding is that already for small values of $k$, say $k=3$, there is almost no difference anymore between the efficiency of Tyler's M and the $k$-step SCM, over almost the complete range of possible values for $\gamma$. For instance, for $\gamma=0.001, \operatorname{Eff}_{3}\left(F_{\gamma}\right)$ still equals 0.44 . Hence the loss in efficiency of using the $k=3$ version instead of the fully iterated Tyler's M-estimator is only important for extremely small values of $\gamma$.

\section{Simulations}

In this section we perform a modest simulation study to confirm the asymptotic efficiencies obtained in the previous Section. We generate $m=10000$ samples from a bivariate normal distribution with covariance matrix $\Sigma_{\gamma}$, as in (12).

For every generated sample, we compute the first eigenvector $\hat{v}_{1}^{j}$ of the multivariate scale estimator, and summarize the outcomes by the

$$
\operatorname{MSE}\left(\hat{v}_{1}\right)=\frac{1}{m} \sum_{j=1}^{m}\left(\arccos \left\{\left|v_{1}^{t} \hat{v}_{1}^{j}\right|\right\}\right)^{2}
$$


Table 1 Finite sample efficiencies, over 10,000 simulation runs, of the first eigenvector estimates, for samples of size $n=20,50,100,200,1,000$, generated from a bivariate normal distribution with mean 0 and covariance matrix $\Sigma=\operatorname{diag}(1, \gamma)$, for $\gamma=0.1$ and $\gamma=0.7$. We considered the SCM, and its $k-$ step version. The column with $k=\infty$ corresponds to Tyler's M-estimator

\begin{tabular}{lllllll}
\hline$n$ & SCM & $k=1$ & $k=2$ & $k=3$ & $k=4$ & $k=\infty$ \\
\hline$\gamma=0.1$ & & & & & & \\
20 & 0.338 & 0.382 & 0.404 & 0.411 & 0.414 & 0.415 \\
50 & 0.359 & 0.421 & 0.453 & 0.467 & 0.473 & 0.476 \\
100 & 0.365 & 0.433 & 0.467 & 0.482 & 0.489 & 0.492 \\
200 & 0.363 & 0.430 & 0.463 & 0.477 & 0.483 & 0.495 \\
1000 & 0.361 & 0.435 & 0.474 & 0.493 & 0.501 & 0.504 \\
$\infty$ & 0.365 & 0.436 & 0.473 & 0.489 & 0.496 & 0.500 \\
$\gamma=0.7$ & & & & & & \\
20 & 0.791 & 0.790 & 0.789 & 0.788 & 0.788 & 0.786 \\
50 & 0.655 & 0.655 & 0.655 & 0.654 & 0.654 & 0.653 \\
100 & 0.523 & 0.523 & 0.522 & 0.522 & 0.521 & 0.521 \\
200 & 0.430 & 0.431 & 0.431 & 0.430 & 0.430 & 0.430 \\
1000 & 0.471 & 0.473 & 0.474 & 0.474 & 0.474 & 0.474 \\
$\infty$ & 0.496 & 0.498 & 0.499 & 0.500 & 0.500 & 0.500 \\
\hline
\end{tabular}

with $v_{1}=(1,0)^{t}$ the true eigenvector. This MSE converges to the asymptotic variance (16) introduced in Sect. 3, see Croux et al. (2002). Dividing the MSE obtained using the sample covariance matrix by the MSE resulting from the $k$-step SCM yields the finite sample counterpart of (17). Finite sample efficiencies are obtained for the SCM, its $k$-step version, with $k=1,2,3,4$, and for the fully iterated version $(k=\infty)$, i.e. Tyler's M-estimator. We consider sample sizes $n=20,50,100,200,1,000$. Furthermore, we take $\gamma=0.1$, where the deviation of sphericity is very strong, and $\gamma=0.7$, where we are closer to a spherical model distribution. The standard errors around the results reported in Table 1 are at most 0.02 .

We can see from Table 1 that the finite sample efficiencies converge to their asymptotic counterparts $(n=\infty)$, although not monotonically. Note that for $\gamma=0.7$ and $n=20,50$ the finite sample efficiencies are considerably higher than expected. When we are closer to the spherical model distribution $(\gamma=0.7)$, there appears to be no significant difference between the efficiency of the different $k$-step estimators and Tyler's M.

\section{Conclusion}

The use of $k$-step estimators is widespread in the statistical literature [see Hallin et al. (2006) for a recent contribution]. Starting from an initial estimate, one makes sequential updates of the estimator, resulting in a sequence of $k$-step estimators. The initial estimator is consistent, and the $k$-step versions increase the efficiency. Most often, the initial estimator is easy to compute, and the $k$-step updates come with almost no 
additional computational effort. As such, Taskinen et al. (2010) use Tyler's M-estimator, or its symmetrized version, as a starting estimator, and increase its efficiency by carrying out $k$-steps. However, since this starting estimator has a low breakdown point, the same holds for the $k$-step improvement. Our approach is different, we start from a high breakdown estimator and all further $k$-step SCM inherit this high breakdown point.

As we showed in Sect. 3, the efficiency of the $k$-step SCM estimator increases quickly to $p /(p+2)$, the efficiency of Tyler's M-estimator. To increase further the efficiency, additional weights can be added, resulting in

$$
\hat{\Sigma}_{k}(X)=\frac{1}{n} \sum_{i=1}^{n} \frac{\left(X_{i}-\hat{\mu}_{n}\right)\left(X_{i}-\hat{\mu}_{n}\right)^{t}}{\left(X_{i}-\hat{\mu}_{n}\right)^{t} \hat{\Sigma}_{k-1}^{-1}\left(X_{i}-\hat{\mu}_{n}\right)} w\left(\left(X_{i}-\hat{\mu}_{n}\right)^{t} \hat{\Sigma}_{k-1}^{-1}\left(X_{i}-\hat{\mu}_{n}\right)\right)
$$

for $k \geq 1$. Kent and Tyler (1991) show that this sequence of estimators converges to an M-estimator of shape if the weight function $w$ is strictly increasing. Using a bounded weight function will retain the breakdown point of the initial SCM. Assume that we take a weight function $w$ that is bounded, strictly increasing, and such that $w(s)=s$ for $s \leq c$. For $c$ and $k$ large enough, this results in a high breakdown point estimator having a Gaussian efficiency arbitrarily close to $100 \%$.

Our paper has several limitations. In this paper we keep the location estimator fixed. One could consider to update the location estimator as well, as in Hettmansperger and Randles (2002). We claim that the $k$-step spatial median retains the maximal breakdown point of the $L_{1}$-median, and that updating the location estimator will not change the efficiency of the $k$-step SCM estimator, neither its breakdown point.

Another limitation is that the $k$-step SCM estimator is only consistently estimating the orientation of the scatter matrix. However, this is the most crucial part, since the eigenvalues of $\Sigma$ can be estimated afterwards by applying an efficient and robust scale estimator to the data projected on the respective eigenvectors. Finally, we only measure robustness by means of the breakdown point. It would be of interest to consider also the maxbias curve, as was done by Rousseeuw and Croux (1994) for univariate $k$-step M-estimators.

Open Access This article is distributed under the terms of the Creative Commons Attribution Noncommercial License which permits any noncommercial use, distribution, and reproduction in any medium, provided the original author(s) and source are credited.

\section{Appendix}

Proof of proposition 1 Denote $\hat{\Sigma}=\hat{\Sigma}_{S}$. We first show that $\varepsilon^{*}(\hat{\Sigma}, X) \geq\left\lfloor\frac{n+1}{2}\right\rfloor / n$. Let $m<\left\lfloor\frac{n+1}{2}\right\rfloor$ and replace $m$ observations of $X$ to get $X^{\prime}=\left\{x_{1}, \ldots, x_{n-m}\right.$, $\left.x_{n-m+1}^{\prime}, \ldots, x_{n}^{\prime}\right\}$. Without loss of generality, we assume that the first $n-m$ values of $X$ remain unchanged. Let $\hat{\mu}^{\prime}$ be the $L_{1}$-median computed from $X^{\prime}$. We need to show that there exist constants $\delta_{X}, N_{X}>0$ such that $\lambda_{\min }\left(\hat{\Sigma}\left(X^{\prime}\right)\right)>\delta_{X}$ and $\lambda_{\max }\left(\hat{\Sigma}\left(X^{\prime}\right)<N_{X}\right.$. We have 


$$
\begin{aligned}
\lambda_{\max }\left(\hat{\Sigma}\left(X^{\prime}\right)\right) & =\sup _{\|u\|=1} u^{t} \hat{\Sigma}\left(X^{\prime}\right) u \\
& =\sup _{\|u\|=1} \frac{1}{n} \sum_{i=1}^{n} \frac{u^{t}\left(x_{i}^{\prime}-\hat{\mu}^{\prime}\right)\left(x_{i}^{\prime}-\hat{\mu}^{\prime}\right)^{t} u}{\left\|x_{i}^{\prime}-\hat{\mu}^{\prime}\right\|^{2}} \\
& =\sup _{\|u\|=1} \frac{1}{n} \sum_{i=1}^{n} \frac{\left(u^{t}\left(x_{i}^{\prime}-\hat{\mu}^{\prime}\right)\right)^{2}}{\left\|x_{i}^{\prime}-\hat{\mu}^{\prime}\right\|^{2}} \\
& \leq \sup _{\|u\|=1} \frac{1}{n} \sum_{i=1}^{n} \frac{\|u\|^{2}\left\|x_{i}^{\prime}-\hat{\mu}^{\prime}\right\|^{2}}{\left\|x_{i}^{\prime}-\hat{\mu}^{\prime}\right\|^{2}}=1=N_{X} .
\end{aligned}
$$

For every subset $J$ of size $n-m$ from $\{1, \ldots, n\}$, define $\eta_{J}=\max _{i \in J} d^{2}\left(x_{i}, H_{J}\right)$, where $H_{J}$ is the hyperplane minimizing $\sum_{i \in J} d^{2}\left(x_{i}, H\right)$ over all possible hyperplanes $H$, and $d(x, H)$ is the Euclidean distance between an observation $x$ and a hyperplane $H$. Define then $\eta_{X}=\min _{J} \eta_{J}$. Since $n-m \geq\left\lfloor\frac{n+1}{2}\right\rfloor$, and no $\left\lfloor\frac{n+1}{2}\right\rfloor$ original observations are on the same hyperplane, we have that $\eta_{X}>0$. Furthermore, since there is not yet breakdown of the $L_{1}$-median, there exists a constant $\bar{M}$ such that $\left\|\hat{\mu}^{\prime}\right\| \leq \bar{M}_{X}$. Finally, let $M_{X}=\max _{1 \leq i \leq n}\left\|x_{i}\right\|$, and $\delta_{X}=0.5 \eta_{X} /\left(n\left(M_{X}+\bar{M}_{X}\right)^{2}\right)$. Using all these notations, we obtain

$$
\begin{aligned}
\lambda_{\min }\left(\hat{\Sigma}, X^{\prime}\right) & =\min _{\|u\|=1} u^{t} \hat{\Sigma}\left(X^{\prime}\right) u \\
& =\min _{\|u\|=1} \frac{1}{n} \sum_{i=1}^{n} \frac{u^{t}\left(x_{i}^{\prime}-\hat{\mu}^{\prime}\right)\left(x_{i}^{\prime}-\hat{\mu}^{\prime}\right)^{t} u}{\left\|x_{i}^{\prime}-\hat{\mu}^{\prime}\right\|^{2}} \\
& =\min _{\|u\|=1} \frac{1}{n} \sum_{i=1}^{n}\left[\frac{u^{t}\left(x_{i}^{\prime}-\hat{\mu}^{\prime}\right)}{\left\|x_{i}^{\prime}-\hat{\mu}^{\prime}\right\|^{2}}\right]^{2} \\
& \geq \min _{\|u\|=1} \frac{1}{n} \sum_{i=1}^{n-m}\left[\frac{u^{t}\left(x_{i}-\hat{\mu}^{\prime}\right)}{\left\|x_{i}-\hat{\mu}^{\prime}\right\|^{2}}\right]^{2} \\
& \geq \min _{\|u\|=1} \sum_{i=1}^{n-m}\left[u^{t}\left(x_{i}-\hat{\mu}^{\prime}\right)\right]^{2} \frac{1}{n\left(M_{X}+\bar{M}_{X}\right)^{2}} \\
& \geq \sum_{i=1}^{n-m} d^{2}\left(x_{i}, H_{\{1, \ldots, n-m\}}\right) \frac{1}{n\left(M_{X}+\bar{M}_{X}\right)^{2}} \\
& \geq \eta_{X} \frac{1}{n\left(M_{X}+\bar{M}_{X}\right)^{2}}=\delta_{X} .
\end{aligned}
$$

Now we will show that $\varepsilon^{*}\left(\Sigma_{S}, X\right) \leq\left[\frac{n+1}{2}\right] / n$. We replace $m=\left[\frac{n+1}{2}\right]$ observations of $X$ to a constant vector $\tilde{x}$. Since the $L_{1}$-median has the multivariate exact fit property (Maronna et al. 2006), we have $\hat{\mu}^{\prime}=\tilde{x}$. we take $\|\tilde{x}\|>M_{X}$, such that $\left\|x_{i}-\tilde{x}\right\| \geq\|\tilde{x}\|-\left\|x_{i}\right\| \geq\|\tilde{x}\|-M_{X}$, for every index $i$. Let $\tilde{u}$ a vector of norm one, orthogonal to $\tilde{x}$, then 


$$
\begin{aligned}
& \lambda_{\text {inf }}\left(\hat{\Sigma}\left(X^{\prime}\right)\right)=\inf _{\|u\|=1} \frac{1}{n} \sum_{i=1}^{n-m-1}\left[\frac{u^{t}\left(x_{i}-\tilde{x}\right)}{\left\|x_{i}-\tilde{x}\right\|}\right]^{2} \\
& \leq \frac{1}{n} \sum_{i=1}^{n-m} \frac{\left(\tilde{u}^{t} x_{i}\right)^{2}}{\left\|x_{i}-\tilde{x}\right\|^{2}} \\
& \leq \frac{1}{n} \sum_{i=1}^{n-m} \frac{\left\|x_{i}\right\|^{2}}{\left\|x_{i}-\tilde{x}\right\|^{2}} \\
& \leq M_{X}^{2} \frac{1}{n} \sum_{i=1}^{n-m} \frac{1}{\left\|x_{i}-\tilde{x}\right\|^{2}} \\
& \leq \frac{n-m}{n} \frac{M_{X}^{2}}{\left(\|\tilde{x}\|-M_{X}\right)^{2}},
\end{aligned}
$$

which tends to 0 when $\|\tilde{x}\|$ is tending to infinity.

\section{References}

Bensmail H, Celeux G (1996) Regularized Gaussian discriminant analysis through eigenvalue decomposition. J Am Stat Assoc 91:1743-1749

Croux C, Haesbroeck G (2000) Principal component analysis based on robust estimators of the covariance or correlation matrix: influence functions and efficiencies. Biometrika 87:603-618

Croux C, Ollila E, Oja H (2002) Sign and rank covariance matrices: statistical properties and application to principal components analysis. In: Dodge Y (ed) Statistical data analysis based on the L1-norm and related methods. Birkhauser, Basel, pp 257-271

Dümbgen L, Tyler DE (2005) On the breakdown properties of some multivariate M-functionals. Scand J Stat 32:247-264

Frahm G (2009) Asymptotic distiributions of robust shape matrices and scale. J Multivar Anal 100:13291337

Hallin M, Oja H, Paindaveine D (2006) Semiparametrically efficient rank-based inference for shape. II. Optimal R-estimation of shape. Ann Stat 34:2757-2789

Hettmansperger TP, Randles RH (2002) A practical affine equivariant multivariate médian. Biometrika 89(4):851-860

Kent JT, Tyler DE (1991) Redescending M-estimates of multivariate location and scatter. Ann Stat 19:21022119

Locantore N, Marron JS, Simpson DG, Tripoli N, Zhang JT, Cohen KL (1999) Robust principal components for functional data. Test $8: 1-28$

Lopuhäa HP, Rousseeuw PJ (1991) Breakdown points of affine equivariant estimators of multivariate location and covariance matrices. Ann Stat 19:229-248

Maronna R, Martin D, Yohai V (2006) Robust statistics. Wiley, New York

Oja H (2010) Multivariate nonparametric methods with R. An approach based on spatial signs and ranks, Springer, Berlin (in press)

Ollila E, Heetmansperger TP, Oja H (2002) Affine equivariant multivariate sign methods (unpublished)

Paindaveine D (2008) A canonical definition of shape. Stat Probab Lett 78:2240-2247

Rousseeuw PJ, Croux C (1994) The bias of k-step M-estimators. Stat Probab Lett 20:411-420

Sirkia S, Taskinen S, Oja H, Tyler D (2009) Tests and estimates of shape based on spatial signs and ranks. J Nonparametr Stat 21:15-176

Taskinen S, Sirkiä S, Oja H (2010) k-step Shape estimators based on spatial signs and ranks. J Stat Plan Inference (in press)

Tyler DE (1987) A distribution-free M-estimator of multivariate scatter. Ann Stat 15:234-251 
Visuri S, Ollila E, Koivunen V, Möttönen J, Oja H (2003) Affine equivariant multivariate rank methods. J Stat Plan Infer 114:161-185

Yadine A (2006) Robustness and efficiency of multivariate scatter estimators", Ph.D. dissertation, Université Libre de Bruxelles, Bruxelles 